\title{
RICCATI INEQUALITY AND OSCILLATION CRITERIA FOR PDE WITH $p$-LAPLACIAN
}

\author{
ZHITING XU
}

Received 1 November 2003; Revised 25 December 2004; Accepted 25 December 2004

Oscillation criteria for PDE with $p$-Laplacian $\operatorname{div}\left(A(x)\|D u\| p^{p-2} D u\right)+p(x)|u|^{p-2} u=0$ are obtained via Riccati inequality. Some of them are extensions of the results for the second-order linear ODE to this equation.

Copyright (c) 2006 Zhiting Xu. This is an open access article distributed under the Creative Commons Attribution License, which permits unrestricted use, distribution, and reproduction in any medium, provided the original work is properly cited.

\section{Introduction}

In this paper, we are interested in obtaining oscillation criteria for the solutions of the second-order partial differential equation (PDE) with $p$-Laplacian

$$
\operatorname{div}\left(A(x)\|D u\|^{p-2} D u\right)+p(x)|u|^{p-2} u=0
$$

in the exterior domain $\Omega(1):=\left\{x \in \mathbb{R}^{N}:\|x\|>1\right\}$, where $p>1, x=\left(x_{1}, \ldots, x_{N}\right) \in \mathbb{R}^{N}$, $N \geq 2, D u=\left(\partial u / \partial x_{1}, \ldots, \partial u / \partial x_{N}\right),\|x\|$ is usual Euclidean norm in $\mathbb{R}^{N}$.

Throughout this paper we will assume that

$\left(\mathrm{A}_{1}\right) p \in C_{\mathrm{loc}}^{\mu}(\Omega(1)), 0<\mu<1$, and $p>1$ constant,

$\left(\mathrm{A}_{2}\right) A=\left(A_{i j}(x)\right)_{N \times N}$ is a real symmetric positive define matrix function with $A_{i j} \in$ $C_{\mathrm{loc}}^{1+\mu}(\Omega(1)), i, j=1, \ldots, N$, and $0<\mu<1$.

Denote by $\lambda_{\min }(x)$ the smallest eigenvalue of $A$. We suppose that there is a function $\lambda \in C\left([1, \infty), \mathbb{R}^{+}\right)$such that

$$
\min _{|x|=r} \frac{\lambda_{\min }(x)}{\|A\|^{q}} \geq \lambda(r) \quad \text { for } r>1,
$$

where $\|A\|$ denotes the norm of the matrix $A$, that is, $\|A\|=\left[\sum_{i, j=1}^{N} A_{i j}^{2}(x)\right]^{1 / 2}$, and $q$ is the conjugate number to $p$, that is, $q=p /(p-1)$.

By a solution of (1.1), we mean a function $u(x) \in C_{\mathrm{loc}}^{2+\mu}(\Omega(1))$ which satisfies (1.1) almost every on $\Omega(1)$. We restrict our attention to the nontrivial solution $u(x)$ of (1.1), that is, to the solution $u(x)$ such that $\sup _{x \in \Omega(1)}\{|u(x)|\}>0$. A nontrivial solution of (1.1) 
is said to be oscillatory if $u$ has zero on $\Omega(a)$ for every $a>1$. Equation (1.1) is said to be oscillatory if every solution (if any exists) is oscillatory. Conversely, (1.1) is nonoscillatory if there exists a solution which is not oscillatory.

PDE with $p$-Laplacian have wide applications in various physical and biological problems. These equations describe the behavior of the systems whose energetic functional is of degree $p$. Further let us mention the equation

$$
\operatorname{div}\left(\|D u\|^{p-2} D u\right)+\lambda u=0
$$

which appears in the study of non-Newtonian fluids. (Non-Newtonian fluids are typically the fluids which are a suspension of particles, deformed by the acting of forces, in liquid. Most biological fluids, like blood, have this property.) Other relevant applications of the equations with $p$-Laplacian are in the glaciology and slow diffusion problems (the flow through porous media, e.g., a dam filled by rocks). For more examples of applications the reader is referred to [1] and the references therein.

In the qualitative theory of nonlinear partial differential equation, one of the important problems is to determine whether or not solutions of the equation under consideration are oscillatory. For the quasilinear elliptic equation

$$
\operatorname{div}\left(\|D u\|^{p-2} D u\right)+p(x)|u|^{p-2} u=0 .
$$

Recently, taking advantage of the oscillation analysis of the second-order linear ordinary differential equation (see, $[3,5,6]$ )

$$
\left(r(t) y^{\prime}(t)\right)^{\prime}+p(t) y(t)=0
$$

Usami [4], Xu and Xing [7] have established some oscillation criteria for (1.4). In this paper, we will continue in this direction and study the oscillatory properties of the general equation (1.1). By using the generalized Riccati inequality established in Section 2 (Lemma 2.1), we try to extend the results in $[2,5]$ to $(1.1)$, which include and improve the results of Usami [4]. We are especially interested in the case where $p(x)$ has a variable sign on $\Omega(1)$.

\section{Preliminaries}

In the sequel, we say that a function $H=H(r, s)$ belongs to a function class $\mathscr{H}$, denoted by $H \in \mathscr{H}$, if $H \in C(D,[0, \infty))$, where $D=\{(r, s):-\infty<s \leq r<\infty\}$, which satisfies

$$
H(r, r)=0, \quad H(r, s)>0 \quad \text { for } r>s,
$$

and has partial derivatives $\partial H / \partial r$ and $\partial H / \partial s$ on $D$ such that

$$
\frac{\partial H}{\partial r}=h_{1}(r, s) H(r, s), \quad \frac{\partial H}{\partial s}=-h_{2}(r, s) H(r, s),
$$

where $h_{1}, h_{2} \in L_{\mathrm{loc}}(D, \mathbb{R})$. 
Let any functions $\rho \in C\left([1, \infty), \mathbb{R}^{+}\right)$and $\kappa \in C([1, \infty), \mathbb{R})$, we take two integral operators $X_{\tau}^{\rho}$ and $Y_{\tau}^{\rho}$, which are define in [6], in terms of $H(r, s)$ and $\rho(s)$ as

$$
\begin{aligned}
& X_{\tau}^{\rho}(\kappa ; r)=\int_{\tau}^{r} H(s, \tau) \kappa(s) \rho(s) d s, \quad r \geq \tau \geq 1, \\
& Y_{\tau}^{\rho}(\kappa ; r)=\int_{\tau}^{r} H(r, s) \kappa(s) \rho(s) d s, \quad r \geq \tau \geq 1 .
\end{aligned}
$$

To formulate our results, we will the following notations. For a given function $\varphi \in$ $C\left(\mathbb{R}^{+}, \mathbb{R}^{+}\right)$, we define

$$
\begin{aligned}
g(r) & =\frac{p}{2} \lambda(r)\left[\omega r^{N-1} \varphi(r)\right]^{1-q}, \\
\Theta(r) & =\varphi(r) \int_{S_{r}} p(x) d \sigma-\frac{1}{p^{1-p}} g^{1-r)}\left|\frac{\varphi^{\prime}(r)}{\varphi(r)}\right|^{p},
\end{aligned}
$$

where $S_{r}=\left\{x \in R^{N}:\|x\|=r\right\}, d \sigma$ denotes the spherical integral element in $R^{N}$, and $\omega$ represents the surface measure of unit sphere.

In what follows, we will prove three lemmas, which will be useful for establishing oscillation criteria for (1.1). The first is modified version of [4, Lemma 1]. The second and the third are the direct extensions of [2, Lemmas 2.2 and 2.3] to the case of (1.1).

LEMma 2.1. Let $u=u(x)$ be a nonoscillatory solution of (1.1). For an arbitrary given function $\varphi \in C^{1}\left(\mathbb{R}^{+}, \mathbb{R}^{+}\right)$, then the function $Z(r)$ defined by

$$
Z(r)=\varphi(r) \int_{S_{r}} W(x) \cdot \nu(x) d \sigma \quad \text { for } r \geq a \geq 1
$$

satisfies the generalized Riccati inequality

$$
Z^{\prime}(r) \leq-\Theta(r)-\frac{1}{q} g(r)|Z(r)|^{q} \text { for } r \geq a,
$$

where

$$
W(x)=\frac{\|D u\|^{p-2} A(x) D u}{|u|^{p-2} u}
$$

and $\nu(x)=x /\|x\|,\|x\| \neq 0$, denotes the outward unit normal.

Proof. Without loss of generality, we may assume that $u=u(x)>0$ for $\|x\| \geq a \geq 1$. In view of (1.1), a directly computation yields that

$$
\operatorname{div} W(x)=-p(x)-(p-1) u^{-p}\|D u\|^{p-2}(D u)^{T} A(x) D u .
$$

Since

$$
\|W(x)\| \leq u^{1-p}\|D u\|^{p-1}\|A\|
$$


4 Riccati inequality and oscillation criteria

then

$$
\begin{aligned}
& \|D u\|^{p-2}(D u)^{T} A(x) D u \\
& \quad \geq \lambda_{\min }(x)\|D u\|^{p} \geq u^{p} \lambda_{\min }(x)\left[\frac{\|W(x)\|}{\|A\|}\right]^{q} \geq u^{p} \lambda(\|x\|)\|W(x)\|^{q} .
\end{aligned}
$$

Using Green's formula in (2.5), observing (2.8) and (2.10), we have

$$
\begin{aligned}
Z^{\prime}(r) & =\frac{\varphi^{\prime}(r)}{\varphi(r)} Z(r)+\varphi(r) \int_{S_{r}} \operatorname{div} W(x) d \sigma \\
& \leq \frac{\varphi^{\prime}(r)}{\varphi(r)} Z(r)-\varphi(r)\left[\int_{S_{r}} p(x) d \sigma+(p-1) \lambda(r) \int_{S_{r}}\|W(x)\|^{q} d \sigma\right] .
\end{aligned}
$$

By Hölder's inequality

$$
\begin{aligned}
\left|\int_{S_{r}} W(x) \cdot v(x) d \sigma\right| & \leq\left[\int_{S_{r}}\|\nu(x)\|^{p} d \sigma\right]^{1 / p}\left[\int_{S_{r}}\|W(x)\|^{q} d \sigma\right]^{1 / q} \\
& =\left[\omega r^{N-1}\right]^{1 / p}\left[\int_{S_{r}}\|W(x)\|^{q} d \sigma\right]^{1 / q},
\end{aligned}
$$

and by Young's inequality

$$
\left|\frac{\varphi^{\prime}(r)}{\varphi(r)} Z(r)\right| \leq \frac{1}{p^{1}} g^{1-p}(r)\left|\frac{\varphi^{\prime}(r)}{\varphi(r)}\right|^{p}+\frac{1}{q} g(r)|Z(r)|^{q} .
$$

Thus, inequality (2.6) follows from (2.11)-(2.13). The proof is complete.

Lemma 2.2. Let $u=u(x)$ be a solution of (1.1) such that $u(x) \neq 0$ for $\|x\| \in(a, c]$. For any $\varphi \in C^{1}\left([1, \infty), \mathbb{R}^{+}\right)$, let $Z(r)$ be defined on $(a, c]$ by $(2.5)$. Then, for any $H \in \mathcal{H}$ and $\rho \in C^{1}\left([1, \infty), \mathbb{R}^{+}\right)$, we have

$$
X_{a}^{\rho}\left(\Theta-\frac{1}{p} g^{1-p}\left|h_{1}+\frac{\rho^{\prime}}{\rho}\right|^{p} ; c\right) \leq-H(c, a) \rho(c) Z(c) .
$$

Proof. By Lemma 2.1, we have that, for $s \in(a, c]$,

$$
\Theta(s) \leq-Z^{\prime}(s)-\frac{1}{q} g(s)|Z(s)|^{q} .
$$

Applying the operator $X_{r}^{\rho}(a \leq r \leq c)$ to (2.15), using (2.1) and (2.2), we find

$$
X_{r}^{\rho}(\Theta ; c) \leq-H(c, r) \rho(c) Z(c)+X_{c}^{\rho}\left(\left|h_{1}+\frac{\rho^{\prime}}{\rho}\right||Z| ; c\right)-X_{r}^{\rho}\left(\frac{1}{q} g|Z|^{q} ; c\right) .
$$

According to Young's inequality

$$
\left|h_{1}+\frac{\rho^{\prime}}{\rho}\right||Z| \leq \frac{1}{p} g^{1-p}\left|h_{1}+\frac{\rho^{\prime}}{\rho}\right|^{p}+\frac{1}{q} g|Z|^{q},
$$


then, (2.16) can be simplified to

$$
X_{r}^{\rho}\left(\Theta-\frac{1}{p} g^{1-p}\left|h_{1}+\frac{\rho^{\prime}}{\rho}\right|^{p} ; c\right) \leq-H(c, r) \rho(c) Z(c) .
$$

Letting $r \rightarrow a^{+}$in the above, we obtain (2.14). The proof is complete.

Similarly as in the proof Lemma 2.2, we have the following lemma.

LEMMA 2.3. Let $u=u(x)$ be a solution of (1.1) such that $u(x) \neq 0$ for $\|x\| \in[c, b)$. For any $\varphi \in C^{1}\left([1, \infty), \mathbb{R}^{+}\right)$, let $Z(r)$ be defined on $[c, b)$ by (2.5). Then, for any $H \in \mathcal{H}$ and $\rho \in C^{1}\left([1, \infty), \mathbb{R}^{+}\right)$, we have

$$
Y_{c}^{\rho}\left(\Theta-\frac{1}{p} g^{1-p}\left|h_{2}-\frac{\rho^{\prime}}{\rho}\right|^{p} ; b\right) \leq H(b, c) \rho(c) Z(c) .
$$

\section{Main results}

The first theorem presents an oscillation criterion for (1.1) which is an analogue of Wintner's criterion [5] for (1.5).

Theorem 3.1. Assume that there exist functions $\varphi, \rho \in C^{1}\left([1, \infty), \mathbb{R}^{+}\right)$such that

$$
\begin{gathered}
\int^{\infty}\left|\rho^{\prime}(s)\right|^{p}[\rho(s) g(s)]^{1-p} d s<\infty, \\
\int^{\infty} \rho(s) \Theta(s) d s=\infty \\
\int^{\infty} g(s) \rho^{1-q}(s) d s=\infty
\end{gathered}
$$

then (1.1) is oscillatory.

Proof. Let $u=u(x)$ be a nonoscillatory solution of (1.1). Without loss of generality let us consider that $u=u(x)>0$ for $\|x\| \geq l$ for some sufficient large $l>1$. Let $Z(r)$ be well defined on $[l, \infty)$ by $(2.5)$, from Lemma 2.1 , we have that

$$
Z^{\prime}(r)+\Theta(r)+\frac{1}{q} g(r)|Z(r)|^{q} \leq 0 \quad \text { for } r \geq l .
$$

Multiplying (3.4) by $\rho(s)$, and integrating both sides of the results on $[l, r]$, we have

$$
\rho(r) Z(r)+\int_{l}^{r} \rho(s) \Theta(s) d s+\frac{1}{q} \int_{l}^{r} \rho(s) g(s)|Z(r)|^{q} d s \leq C_{1}+\int_{l}^{r} \rho^{\prime}(s) Z(s) d s,
$$

where $C_{1}$ is a constant. Hölder's inequality gives

$$
\begin{aligned}
\int_{l}^{r}\left|\rho^{\prime}(s) Z(s)\right| d s & \leq\left[\int_{l}^{r}\left|\rho^{\prime}(s)\right|^{p}[\rho(s) g(s)]^{1-p} d s\right]^{1 / p}\left[\int_{l}^{r} \rho(s) g(s)|Z(s)|^{q} d s\right]^{1 / q} \\
& \leq C_{2}\left[\int_{l}^{r} \rho(s) g(s)|Z(s)|^{q} d s\right]^{1 / q},
\end{aligned}
$$


6 Riccati inequality and oscillation criteria

where $C_{2}=\int^{\infty}\left|\rho^{\prime}(s)\right|^{p}[\rho(s) g(s)]^{1-p} d s$ is finite by (3.1). Thus, by (3.5) and (3.6), we have

$$
\rho(r) Z(r)+\int_{l}^{r} \rho(s) \Theta(s) d s+\frac{1}{q} H(r)-C_{2} H^{1 / q}(r) \leq C_{1} \quad \text { for } r \geq l,
$$

where

$$
H(r)=\int_{l}^{r} \rho(s) g(s)|Z(s)|^{q} d s
$$

Note that for $q \geq 1$, the function $1 /(2 q) H(r)-C_{2} H^{1 / q}(r)$ is bounded from below on $[0, \infty)$. By (3.2), (3.7) implies that

$$
\rho(r) Z(r) \leq-\frac{1}{2 q} H(r) \quad \text { for some sufficiently large } l_{1} \geq l .
$$

So

$$
|Z(r)| \geq \frac{1}{2 q} \frac{1}{\rho(r)} H(r) \quad \text { for } r \geq l_{1} .
$$

Thus, we obtain, by (3.10)

$$
H^{\prime}(r)=\rho(r) g(r)|Z(r)|^{q}=\frac{1}{(2 q)^{q}} g(r) \rho^{1-q}(r) H^{q}(r) \quad \text { for } r \geq l_{1} .
$$

Dividing the both sides by $H^{q}(r)$ and integrating it, we have

$$
\frac{1}{q-1} H^{1-q}\left(l_{1}\right) \geq \frac{1}{(2 q)^{q}} \int_{l_{1}}^{r} g(s) \rho^{1-q}(s) d s
$$

which contradicts (3.3). The proof is complete.

Remark 3.2. Let $\varphi(r) \equiv 1$, Theorem 3.1 improves in [4, Theorem 4] for (1.4).

The next theorem is an immediate consequence of Lemmas 2.2 and 2.3, which provides the domain oscillation criteria for (1.1) and extends [2, Corollary 2.1].

Theorem 3.3. Assume that for some $c \in(a, b)$ and for some $H \in \mathscr{H}, \varphi, \rho \in C^{1}\left([1, \infty), \mathbb{R}^{+}\right)$ such that

$$
\frac{1}{H(c, a)} X_{a}^{\rho}\left(\Theta-\frac{1}{p} g^{1-p}\left|h_{1}+\frac{\rho^{\prime}}{\rho}\right|^{p} ; c\right)+\frac{1}{H(b, c)} Y_{c}^{\rho}\left(\Theta-\frac{1}{p^{1-p}} g^{1-} h_{2}-\left.\frac{\rho^{\prime}}{\rho}\right|^{p} ; b\right)>0,
$$

then every solution $u(x)$ of (1.1) has at least one zero on $\Omega(a, b)=\left\{x \in R^{N}: a<\|x\|<b\right\}$.

Proof. Equation (3.13) implies that both (2.14) and (2.19) do not hold for the given $c$, and hence every solution $u(x)$ of $(1.1)$ has at least one zero in $\Omega(a, b)$. The proof is complete. 
Theorem 3.4. Assume that for some $H \in \mathscr{H}, \varphi, \rho \in C^{1}\left([1, \infty), \mathbb{R}^{+}\right)$such that for each $l \geq 1$

$$
\begin{aligned}
& \limsup _{r \rightarrow \infty} X_{l}^{\rho}\left(\Theta-\frac{1}{p}\left|h_{1}+\frac{\rho^{\prime}}{\rho}\right|^{p} g^{1-p} ; r\right)>0, \\
& \limsup _{r \rightarrow \infty} Y_{l}^{\rho}\left(\Theta-\frac{1}{p}\left|h_{2}-\frac{\rho^{\prime}}{\rho}\right|^{p} g^{1-p} ; r\right)>0,
\end{aligned}
$$

then (1.1) is oscillatory.

Proof. For any $T \geq 1$, let $a=T$. In (3.14), we choose $l=a$. Then there exists $c>a$ such that

$$
X_{a}^{\rho}\left(\Theta-\frac{1}{p^{2}} g^{1-p}\left|h_{1}+\frac{\rho^{\prime}}{\rho}\right|^{p} ; c\right)>0 \text {. }
$$

In (3.15), we choose $l=c$. Then there exists $b>c$ such that

$$
Y_{c}^{\rho}\left(\Theta-\frac{1}{p^{2}} g^{1-p}\left|h_{2}-\frac{\rho^{\prime}}{\rho}\right|^{p} ; b\right)>0 \text {. }
$$

Combining (3.16) and (3.17), we obtain (3.13). The conclusion thus comes from Theorem 3.3. The proof is complete.

Let

$$
H(r, s)=(r-s)^{\alpha}, \quad \rho(s) \equiv 1 \quad \text { for } r \geq s \geq 1,
$$

where $\alpha>1$. Theorem 3.4 reduces to following corollary.

Corollary 3.5. Assume that there exist a function $\varphi \in([1, \infty), \mathbb{R})$ and a constant $\alpha>1$ such that for each $l \geq 1$

$$
\begin{gathered}
\limsup _{r \rightarrow \infty} \int_{l}^{r}\left[(s-l)^{\alpha} \Theta(s)-\frac{\alpha^{p}}{p}(s-l)^{\alpha-p} g^{1-p}(s)\right] d s>0, \\
\limsup _{r \rightarrow \infty} \int_{l}^{r}\left[(r-s)^{\alpha} \Theta(s) d s-\frac{\alpha^{p}}{p}(r-s)^{\alpha-p} g^{1-p}(s)\right] d s>0,
\end{gathered}
$$

then (1.1) is oscillatory.

Let

$$
G(r)=\int_{1}^{r} g(s) d s \quad \text { for } r \geq 1
$$

Then, we have the following. 
8 Riccati inequality and oscillation criteria

Corollary 3.6. Let $G(\infty)=\infty$ and $\alpha>p-1$. Assume that there exists a function $\varphi \in$ $C^{1}\left([1, \infty), \mathbb{R}^{+}\right)$such that for each $l \geq 1$

$$
\begin{gathered}
\limsup _{r \rightarrow \infty} \frac{1}{G^{\alpha-p+1}(r)} \int_{l}^{r}[G(s)-G(l)]^{\alpha} \Theta(s) d s>\frac{\alpha^{p}}{p(\alpha-p+1)}, \\
\limsup _{r \rightarrow \infty} \frac{1}{G^{\alpha-p+1}(r)} \int_{l}^{r}[G(r)-G(s)]^{\alpha} \Theta(s) d s>\frac{\alpha^{p}}{p(\alpha-p+1)},
\end{gathered}
$$

then (1.1) is oscillatory.

Proof. Let

$$
H(r, s)=[G(r)-G(s)]^{\alpha}, \quad \rho(s) \equiv 1 \quad \text { for } r \geq s \geq 1
$$

Then

$$
h_{1}(r, s)=h_{2}(r, s)=\alpha[G(r)-G(s)]^{-1} g(s) .
$$

Noting that

$$
\begin{aligned}
X_{l}^{\rho}\left(g^{1-p}\left|h_{1}+\frac{\rho^{\prime}}{\rho}\right|^{p} ; r\right) & =\alpha^{p} \int_{l}^{r}[G(r)-G(l)]^{\alpha-p} g(s) d s \\
& =\frac{\alpha^{p}}{\alpha-p+1}[G(r)-G(l)]^{\alpha-p+1},
\end{aligned}
$$

from (3.21) and (3.25) we have that

$$
\begin{aligned}
\limsup _{r \rightarrow \infty} & \frac{1}{G^{\alpha-p+1}} X_{l}^{\rho}\left(\Theta-\frac{1}{p^{1-p}}\left|h_{1}+\frac{\rho^{\prime}}{\rho}\right|^{p} ; r\right) \\
\quad= & \limsup _{r \rightarrow \infty}\left\{\frac{1}{G^{\alpha}(r)} \int_{l}^{r}[G(r)-G(l)]^{\alpha} \Theta(s) d s-\frac{\alpha^{p}}{p(\alpha-p+1)}\right\}>0 .
\end{aligned}
$$

It follows that

$$
\limsup _{r \rightarrow \infty} X_{l}^{\rho}\left(\Theta-\frac{1}{p^{1-p}} g^{1-}\left|h_{1}+\frac{\rho^{\prime}}{\rho}\right|^{p} ; r\right)>0,
$$

that is, (3.14) holds. Similarly, (3.22) implies that (3.15) holds. By Theorem 3.4. Equation (1.1) is oscillatory. This completes the proof.

If $p=2$ and $A(x)=I$, then (1.1) reduces to the linear equation

$$
\Delta u+p(x) u=0 .
$$

Let $\varphi(r)=1 /\left(\omega r^{N-1}\right)$, then $g(r)=1 / N$. We have the following corollaries.

Corollary 3.7. Let $\alpha<1$. If

$$
\lim _{r \rightarrow \infty} \int_{1}^{r} s^{\alpha} \Theta_{0}(s) d s=\infty
$$


where

$$
\Theta_{0}(r)=\frac{1}{\omega r^{N-1}} \int_{S_{r}} p(x) d \sigma-\frac{N(N-1)^{2}}{2 r^{2}}
$$

Then (3.28) is oscillatory.

Proof. Note that $\Theta(r)=\Theta_{0}(r)$. Let $\rho(r)=r^{\alpha}$, it is easy to show that all conditions of Theorem 3.1 are satisfied. Thus (3.28) is oscillatory.

Let

$$
H(r, s)=(r-s)^{\alpha}, \quad \rho(s) \equiv 1 \quad \text { for } r \geq s \geq 1,
$$

where $\alpha>1$. Similar to the proof of Corollary 3.6, we can prove the following corollary. Corollary 3.8. Let $\alpha>1$ be a constant. If for all $l \geq 1$

$$
\begin{gathered}
\limsup _{r \rightarrow \infty} \frac{1}{r^{\alpha-1}} \int_{l}^{r}(s-l)^{\alpha} \Theta_{0}(s) d s>\frac{\alpha^{2}}{2(\alpha-1)}, \\
\limsup _{r \rightarrow \infty} \frac{1}{r^{\alpha-1}} \int_{l}^{r}(r-s)^{\alpha} \Theta_{0}(s) d s>\frac{\alpha^{2}}{2(\alpha-1)},
\end{gathered}
$$

where $\Theta_{0}(r)$ is as in Corollary 3.7. Then (3.28) is oscillatory.

Finally, we give two examples to illustrate our results. To the best our knowledge, no previous criteria for oscillation can be applied to these examples.

Example 3.9. Consider the equation

$$
\operatorname{div}\left(A(x)\|D u\|^{p-2} D u\right)+\frac{1+k \sin \|x\|}{\|x\|}|u|^{p-2} u=0, \quad\|x\| \geq 1,
$$

where $p \geq 2, N=2, A(x)=\operatorname{diag}(\|x\|,\|x\|), k \in \mathbb{R}, \lambda(r)=2^{-q / 2} r^{1-q}$.

Let $\varphi(r)=1 /(\omega r)$, then

$$
g(r)=p 2^{-p / 2-1}, \quad \Theta(r)=\frac{1+k \sin r}{r}-\frac{1}{p^{p}} 2^{3 / 2 p-1} r^{1-p}
$$

Let $\rho(r)=1$. It is easy to show that all conditions of Theorem 3.1 are satisfied, hence (3.34) is oscillatory.

Example 3.10. Consider the equation

$$
\Delta u+\frac{\gamma}{\|x\|^{2}} u=0, \quad\|x\| \geq 1
$$

where $N=2$, and

$$
p(x)=\frac{\gamma}{|x|^{2}}, \quad \gamma>\frac{3}{2}, \quad \Theta_{0}(r)=\frac{\gamma-1}{r^{2}} .
$$




\section{Riccati inequality and oscillation criteria}

Note that for $\alpha>1$ and all $l \geq 1$

$$
\limsup _{r \rightarrow \infty} \frac{1}{r^{\alpha-1}} \int_{l}^{r}(s-l)^{\alpha} \Theta_{0}(s) d s=\frac{\gamma-1}{\alpha-1} .
$$

For any $\gamma>3 / 2$, there exists $\alpha>1$ such that $(\gamma-1) /(\alpha-1)>\alpha^{2} /(\alpha-1)$. This means that (3.32) holds. By [2, Lemma 3.1], (3.33) holds for the same $\alpha$. Applying Corollary 3.8, we find that (3.36) is oscillatory for $\gamma>3 / 2$.

\section{References}

[1] J. I. Díaz, Nonlinear Partial Differential Equations and Free Boundaries. Vol. I. Elliptic Equations, Research Notes in Mathematics, vol. 106, Pitman (Advanced Publishing Program), Massachusetts, 1985.

[2] Q. Kong, Interval criteria for oscillation of second-order linear ordinary differential equations, Journal of Mathematical Analysis and Applications 229 (1999), no. 1, 258-270.

[3] Ch. G. Philos, Oscillation theorems for linear differential equations of second order, Archiv der Mathematik. Archives of Mathematics. Archives Mathématiques 53 (1989), no. 5, 482-492.

[4] H. Usami, Some oscillation theorems for a class of quasilinear elliptic equations, Annali di Matematica Pura ed Applicata. Series IV 175 (1998), 277-283.

[5] A. Wintner, A criterion of oscillatory stability, Quarterly of Applied Mathematics 7 (1949), 115117.

[6] J. S. W. Wong, On Kamenev-type oscillation theorems for second-order differential equations with damping, Journal of Mathematical Analysis and Applications 258 (2001), no. 1, 244-257.

[7] Z.-T. Xu and H.-Y. Xing, Oscillation criteria of Kamenev-type for PDE with p-Laplacian, Applied Mathematics and Computation 145 (2003), no. 2-3, 735-745.

Zhiting Xu: Department of Mathematics, South China Normal University,

Guangzhou 510631, China

E-mail address: xztxhyyj@pub.guangzhou.gd.cn 